\title{
On generalized Toeplitz and little Hankel operators on Bergman spaces
}

Article

Accepted Version

Taskinen, J. and Virtanen, J. (2018) On generalized Toeplitz and little Hankel operators on Bergman spaces. Archiv der Mathematik, 110 (2). pp. 155-166. ISSN 1420-8938 doi: https://doi.org/10.1007/s00013-017-1124-2 Available at https://centaur.reading.ac.uk/73602/

It is advisable to refer to the publisher's version if you intend to cite from the work. See Guidance on citing.

To link to this article DOI: http://dx.doi.org/10.1007/s00013-017-1124-2

Publisher: Springer

All outputs in CentAUR are protected by Intellectual Property Rights law, including copyright law. Copyright and IPR is retained by the creators or other copyright holders. Terms and conditions for use of this material are defined in the End User Agreement.

\section{www.reading.ac.uk/centaur}

\section{CentAUR}

Central Archive at the University of Reading

Reading's research outputs online 


\title{
ON GENERALIZED TOEPLITZ AND LITTLE HANKEL OPERATORS ON BERGMAN SPACES
}

\author{
JARI TASKINEN AND JANI VIRTANEN
}

\begin{abstract}
We find a concrete integral formula for the class of generalized Toeplitz operators $T_{a}$ in Bergman spaces $A^{p}, 1<p<\infty$, studied in an earlier work by the authors. The result is extended to little Hankel operators. We give an example of an $L^{2}$-symbol $a$ such that $T_{|a|}$ fails to be bounded in $A^{2}$, although $T_{a}: A^{2} \rightarrow A^{2}$ is seen to be bounded by using the generalized definition. We also confirm that the generalized definition coincides with the classical one whenever the latter makes sense.
\end{abstract}

\section{INTRODUCTION.}

Consider the space $L^{p}:=\left(L^{p}(\mathbb{D}, d A),\|\cdot\|_{p}\right)$, where $1<p<\infty$ and $d A$ is the normalized area measure on the unit disc $\mathbb{D}$ of the complex plane, and the Bergman space $A^{p}$, which is the closed subspace of $L^{p}$ consisting of analytic functions. The Bergman projection $P$ is the orthogonal projection of $L^{2}$ onto $A^{2}$, and it has the integral representation

$$
P f(z)=\int_{\mathbb{D}} \frac{f(\zeta)}{(1-z \bar{\zeta})^{2}} d A(\zeta) .
$$

It is also known to be a bounded projection of $L^{p}$ onto $A^{p}$ for every $1<p<\infty$. For an integrable function $a: \mathbb{D} \rightarrow \mathbb{C}$ and, say, bounded analytic functions $f$, the Toeplitz operator $T_{a}$ with symbol $a$ is defined by

$$
T_{a} f(z)=P(a f)(z)=\int_{\mathbb{D}} \frac{a(\zeta) f(\zeta)}{(1-z \bar{\zeta})^{2}} d A(\zeta) .
$$

Since $P$ is bounded, it follows easily that $T_{a}$ extends to a bounded operator $A^{p} \rightarrow A^{p}$ for $1<p<\infty$, whenever $a$ is a bounded measurable function. The question of the boundedness of $T_{a}$ on $A^{p}$ with unbounded symbols is a long-standing problem. Examples of unbounded symbols inducing bounded Toeplitz operators can be easily constructed, since the behaviour of the symbol inside any compact subset of $\mathbb{D}$ is not important for the boundedness of the operator. Also it is not difficult to find unbounded symbols $a$ for which the integral in (1.1) converges, say, for all $f \in A^{2}$ but the operator is not bounded; see Section 3 for an interesting example. We refer to the papers [1], [2], [3], [4], [5], [6], [8], [10], [11], [12], [14], [15], [16], [18] for classical and recent results on the boundedness and compactness of Toeplitz operators on Bergman spaces.

In the paper [13] we have given a generalized definition of Toeplitz operators, which we denote here $\mathbf{T}_{a}$. The definition takes efficiently into account the possible cancellation phenomena of a symbol. This leads to very weak sufficient conditions for the boundedness of Toeplitz operators. More precisely, in the reference it was

2000 Mathematics Subject Classification. 47B35.

Key words and phrases. Toeplitz operator, little Hankel operator, Bergman space, boundedness, compactness, Fredholm properties. 
shown that $\mathbf{T}_{a}$ is bounded under an averaging condition for the symbol itself rather than for its modulus (the result is repeated and also extended to little Hankel operators in Theorem 1.2, below). However, the presentation of the result in [13] has some shortcomings and accordingly the purpose of this paper is to make some improvements, which will be described in detail at the end of this section.

The results of [13] show that cancellation phenomena may be essential in order to have a bounded operator $T_{a}$. Here, we give an example which emphasizes this: in Section 3 we study the radial symbol $a \in L^{2}$, where $a(z)=|z|^{-1}(1-|z|)^{-1 / 4} \sin ((1-$ $|z|)^{-1}$ ) for $|z| \geq 1 / 2$, and prove that the operator $T_{a}$ is bounded in $A^{p}$, although $T_{|a|}$ is obviously not. Thus, the boundedness of $T_{a}$ cannot be proven by conventional methods that only take into account the modulus of the symbol. We can actually construct such a symbol in any given space $L^{q}$ with $q<\infty$.

Given $a \in L^{1}$, the little Hankel operator $h_{a}$ with symbol $a$ is defined as

$$
h_{a} f(z)=\int_{\mathbb{D}} \frac{a(\zeta) f(\zeta)}{(1-\bar{z} \zeta)^{2}} d A(\zeta)
$$

for $f \in A^{p}$ such that this integral converges. In this paper we make the observation that the generalized definition of a Toeplitz operator and the results of [13] can be extended to the little Hankel case as well. The results for $h_{a}$ are presented in parallel with Toeplitz operators.

As for the notation used in this paper, all function spaces are defined on the open unit disc $\mathbb{D}$. In particular $H^{\infty}$ denotes the Hardy space of bounded analytic functions on $\mathbb{D}$. If $0<\rho<1$, we denote $\mathbb{D}_{\rho}=\{|z| \leq \rho\}$. We also denote the standard weight by $W(z)=1-|z|^{2}$, the kernel functions by $K_{\lambda}(z)=(1-z \bar{\lambda})^{-2}$ and $k_{\lambda}=K_{\lambda} /\left\|K_{\lambda}\right\|_{2}=W(\lambda) k_{\lambda}$, and the Möbius transform by $\varphi_{\lambda}(z)=(\lambda-z) /(1-z \bar{\lambda})$, where $z, \lambda \in \mathbb{D}$. By $C, C^{\prime}$ etc. we mean generic constants, the exact values of which may change from place to place. We will deal with symbols $a$, which always at least belong to the space $L_{\text {loc }}^{1}$ of locally integrable functions on $\mathbb{D}$. For other notation and definitions we refer to the book [17].

Let us first describe briefly the sufficient condition for the boundedness of generalized Toeplitz operators given in [13].

Definition 1.1. Denote by $\mathcal{D}$ the family of the sets $D:=D(r, \theta)$, where

$$
D=\left\{\rho e^{i \phi} \mid r \leq \rho \leq 1-\frac{1}{2}(1-r), \theta \leq \phi \leq \theta+\pi(1-r)\right\}
$$

for all $0<r<1, \theta \in[0,2 \pi]$. We denote $|D|:=\int_{D} d A$ and, for $\zeta=\rho e^{i \phi} \in D(r, \theta)$,

$$
\hat{a}_{D}(\zeta):=\frac{1}{|D|} \int_{r}^{\rho} \int_{\theta}^{\phi} a\left(\varrho e^{i \varphi}\right) \varrho d \varphi d \varrho,
$$

where $a \in L_{\text {loc }}^{1}$. In the following we will study symbols $a$ for which there exists a constant $C>0$ such that

$$
\left|\hat{a}_{D}(\zeta)\right| \leq C
$$

for all $D \in \mathcal{D}$ and all $\zeta \in D$.

It turns out that one can proceed to a generalized definition of bounded Toeplitz operators just by using the condition (1.4). However, for the proofs we need to recall some more definitions from [13]. The countably many sets $D\left(1-2^{-m+1}, 2 \pi(\mu-\right.$ 
1) $\left.2^{-m}\right) \in \mathcal{D}$, where $m \in \mathbb{N}, \mu=1, \ldots, 2^{-m}$, form a decomposition of the disc $\mathbb{D}$. We index these sets somehow into a family $\left(D_{n}\right)_{n=1}^{\infty}$, so that every $D_{n}$ is of the form

$$
D_{n}=\left\{z=r e^{i \theta} \mid r_{n}<r \leq r_{n}^{\prime}, \theta_{n}<\theta \leq \theta_{n}^{\prime}\right\}
$$

where, for some $m$ and $\mu$,

Let $f \in A^{p}$. For all $n=n(m, \mu)$ we write

$$
F_{n} f(z)=\int_{D_{n}} \frac{a(\zeta) f(\zeta)}{(1-z \bar{\zeta})^{2}} d A(\zeta), \quad H_{n} f(z)=\int_{D_{n}} \frac{a(\zeta) f(\zeta)}{(1-\bar{z} \zeta)^{2}} d A(\zeta) \quad \forall z \in \mathbb{D},
$$

so that $F_{n}$ can actually be considered as a conventional, bounded Toeplitz operator on $A^{p}$; similarly for $H_{n}$.

Item $1^{\circ}$ of the following theorem is the main result Theorem 2.3 of [13]. Also, $2^{\circ}$ is an immediate consequence of its proof: we leave to the reader the completely straightforward task to verify that the change $z \bar{\zeta} \rightarrow \bar{z} \zeta$ in the denominator does not affect the proof.

Theorem 1.2. Let $1<p<\infty$ and assume that $a \in L_{\mathrm{loc}}^{1}$ satisfies the condition (1.4). Then, the following hold true.

$1^{\circ}$. Given $f \in A^{p}$, the series $\sum_{n=1}^{\infty} F_{n} f(z)$ converges pointwise, absolutely for almost all $z \in \mathbb{D}$, and the generalized Toeplitz operator $\mathbf{T}_{a}: A^{p} \rightarrow A^{p}$, defined by

$$
\mathbf{T}_{a} f(z)=\sum_{n=1}^{\infty} F_{n} f(z)
$$

is bounded for all $1<p<\infty$, and there is a constant $C$ such that

$$
\left\|\mathbf{T}_{a}\right\| \leq C \sup _{D \in \mathcal{D}, \zeta \in D}\left|\hat{a}_{D}(\zeta)\right| .
$$

$2^{\circ}$. For $f \in A^{p}$, the series $\sum_{n=1}^{\infty} H_{n} f(z)$ converges pointwise, absolutely, for almost all $z \in \mathbb{D}$. We define the generalized little Hankel operator by

$$
\mathbf{h}_{a} f(z)=\sum_{n=1}^{\infty} H_{n} f(z)
$$

Then, $\mathbf{h}_{a}: A^{p} \rightarrow A^{p}$ is bounded for all $1<p<\infty$, and there is a constant $C$ such that

$$
\left\|\mathbf{h}_{a}\right\| \leq C \sup _{D \in \mathcal{D}, \zeta \in D}\left|\hat{a}_{D}(\zeta)\right|
$$

In this paper we improve Theorem 1.2 in the following ways.

$1^{\circ}$. The definition (1.8) of a generalized Toeplitz operator seems to depend on the geometry of a fixed decomposition (1.5) of the unit disc. (No doubt, other decompositions of $\mathbb{D}$, say with different choices of the points $r_{n}$ and $\theta_{n}$, could be used as well, and it is not a priori clear, if the generalized operator defined in that way coincides with (1.8). In fact, an approach using Whitney decompositions with Euclidean rectangles for simply connected domains was presented in [7].) In this paper, formula (2.2), we show that the definition (1.8) coincides with a natural radial limit of conventional Toeplitz operators, and thus the dependence of the definition on the decomposition of the disc vanishes. Moreover, in Corollary 3.1 we show that 
in the case the symbol $a$ and a given $f \in A^{p}$ satisfy $a f \in L^{1}$, then $\mathbf{T}_{a} f$ coincides with the usual integral formula of a Toeplitz operator.

$2^{\circ}$. The terms $F_{n}$ in the series (1.8) are actually conventional, bounded Toeplitz operators. In [13] it is only shown that the series (1.8) converges in the very weak sense mentioned in Theorem 1.2 above. Here, we show in Theorem 2.1 that the operator series $\sum_{n} F_{n}$ converges in the strong operator topology, and the same is true for the new limit representation (2.2). Theorem 2.1 also contains an immediate application of this result to transposed operators.

$3^{\circ}$. The proof of Theorem 2.3 of [13] contains a small error: the inequality (3.8) of the citation is not true as such, since the point $r_{n}^{\prime} e^{i \theta_{n}^{\prime}}$ there is actually on the boundary of the set $D_{n}$. It is however not difficult to fix the flaw, and indeed in the beginning of the proof of Theorem 2.1 we do this by replacing the set $D_{n}$ by a bit larger set denoted by $U_{n}$.

\section{MAin Result.}

We now give a simplified expression of the generalized Toeplitz operator $\mathbf{T}_{a},(1.8)$, and also treat the little Hankel operator as well as the transposed operators. Given $a \in L_{\text {loc }}^{1}$ and $0<\rho<1$ we define the function $a_{\rho}: \mathbb{D} \rightarrow \mathbb{C}$ by $a_{\rho}(z)=a(z)$, if $|z| \leq \rho$ and $a_{\rho}(z)=0$ otherwise. It is plain that the Toeplitz and little Hankel operators

$(2.1) T_{a_{\rho}} f(z)=\int_{\mathbb{D}_{\rho}} \frac{a(\zeta) f(\zeta)}{(1-z \bar{\zeta})^{2}} d A(\zeta), \quad h_{a_{\rho}} f(z)=\int_{\mathbb{D}_{\rho}} \frac{a(\zeta) f(\zeta)}{(1-\bar{z} \zeta)^{2}} d A(\zeta), \quad z \in \mathbb{D}$,

are bounded $A^{p} \rightarrow A^{p}$.

Theorem 2.1. Let $1<p<\infty$ and $1 / p+1 / q=1$, and assume that $a \in L_{\mathrm{loc}}^{1}$ and that (1.4) holds. Then, the generalized Toeplitz operators $\mathbf{T}_{a}: A^{p} \rightarrow A^{p}$ and little Hankel operators $\mathbf{h}_{a}: A^{p} \rightarrow L^{p}$, defined in (1.8) and (1.9), respectively, can be written as

$$
\mathbf{T}_{a} f=\lim _{\rho \rightarrow 1} T_{a_{\rho}} f \quad, \quad \mathbf{h}_{a} f=\lim _{\rho \rightarrow 1} h_{a_{\rho}} f
$$

for all $f \in A^{p}$. The limits converge with respect to the strong operator topology (SOT).

Moreover, the transposed operators (with respect to the standard complex dual pairing) $\mathbf{T}_{a}^{*}: A^{q} \rightarrow A^{q}$ and $\mathbf{h}_{a}^{*}: L^{q} \rightarrow A^{q}$ can be written as

$$
\mathbf{T}_{a}^{*} f=\lim _{\rho \rightarrow 1} T_{\bar{a}_{\rho}} f \quad, \quad \mathbf{h}_{a}^{*} g=\lim _{\rho \rightarrow 1} h_{\bar{a}_{\rho}} f
$$

for $f \in A^{q}$ and $g \in L^{q}$, for almost all $z \in \mathbb{D}$, and the limits here also converge in the SOT.

Remark. In the course of the proof we also show that the sum in (1.8) converges in the SOT and thus improve the result of [13] also in this sense. Of course, the limit on the right hand side of (2.2) cannot in general converge in the operator norm, since the operators $T_{a_{\rho}}$ are compact.

Proof. The proof will be given in a few steps. Moreover, we prove the statement (2.2) only for the Toeplitz operator, but the reader is asked to observe the necessary changes for the little Hankel case.

( $i$ ) In the first step we strengthen the proof of Theorem 2.3 in [13] concerning the sum in (1.8). 
For all $n \in \mathbb{N}$ we define the collection of all sets $D_{\nu}$ which touch the given $D_{n}$, more precisely, $\mathcal{D}_{n}=\left\{D_{\nu}: \nu \in \mathbb{N}, \bar{D}_{\nu} \cap \bar{D}_{n} \neq \emptyset\right\}$. By the definition of the sets $D_{n}$, see (1.2)-(1.6), there exist constants $N, M \in \mathbb{N}$ such that any set $\mathcal{D}_{n}$ contains at most $N$ elements $D_{\nu}$ and on the other hand, any set $D_{\nu}$ belongs to at most $M$ sets $\mathcal{D}_{n}$. Moreover, given $D_{n}$ and $w \in D_{n}$, the subdomain $\cup_{D \in \mathcal{D}_{n}} D=: U_{n}$ always contains a Euclidean disc $D(w, R)$ with center $w$ and radius $R=R(n)>0$ such that $|D(w, R)| \geq C\left|D_{n}\right|$ (use again the choice of the sets $D_{n}$ to see this).

Given an analytic $g: \mathbb{D} \rightarrow \mathbb{C}$, we claim that for each $n$ and $w \in D_{n}$,

$$
|g(w)| \leq \frac{C}{\left|D_{n}\right|} \sum_{D \in \mathcal{D}_{n}} \int_{D}|g(\zeta)| d A(\zeta) .
$$

To prove (2.4), let $D(w, R) \subset U_{n}$ be as above. Then, (2.4) follows from the usual subharmonicity property for $D(w, R)$ :

$$
|g(w)| \leq \frac{C}{|D(w, R)|} \int_{D(w, R)}|g(\zeta)| d A(\zeta) \leq \frac{C^{\prime}}{\left|D_{n}\right|} \int_{U_{n}}|g(\zeta)| d A(\zeta) .
$$

We fix now an arbitrary $f \in A^{p}$. If $n$ is given, we write using a double integration by parts in polar coordinates (see [13] for more details of calculations)

$$
\begin{aligned}
& \int_{D_{n}} \frac{a(\zeta) f(\zeta)}{(1-z \bar{\zeta})^{2}} d A(\zeta)=\left(\int_{r_{n}}^{r_{\theta_{n}}^{\prime}} \int_{\theta_{n}^{\prime}} a\left(\varrho e^{i \varphi}\right) \varrho d \varphi d \varrho\right) \frac{f\left(r_{n}^{\prime} e^{i \theta_{n}^{\prime}}\right)}{\left(1-z r_{n}^{\prime} e^{\left.-i \theta_{n}^{\prime}\right)^{2}}\right.} \\
& -\int_{r_{n}}^{r_{n}^{\prime}}\left(\int_{r_{n}}^{r} \int_{\theta_{n}}^{\theta_{n}^{\prime}} a\left(\varrho e^{i \varphi}\right) \varrho d \varphi d \varrho\right) \partial_{r} \frac{f\left(r e^{i \theta_{n}^{\prime}}\right)}{\left(1-z r e^{-i \theta_{n}^{\prime}}\right)^{2}} d r \\
& -\int_{\theta_{n}}^{\theta_{n}^{\prime}}\left(\int_{r_{n}}^{r_{n}^{\prime}} \int_{\theta_{n}}^{\theta} a\left(\varrho e^{i \varphi}\right) \varrho d \varphi d \varrho\right) \partial_{\theta} \frac{f\left(r_{n}^{\prime} e^{i \theta}\right)}{\left(1-z r_{n}^{\prime} e^{-i \theta}\right)^{2}} d \theta \\
& +\int_{r_{n}}^{r_{n}^{\prime}} \int_{\theta_{n}}^{\theta_{n}^{\prime}}\left(\int_{r_{n}}^{r} \int_{\theta_{n}}^{\theta} a\left(\varrho e^{i \varphi}\right) \varrho d \varphi d \varrho\right) \partial_{r} \partial_{\theta} \frac{f\left(r e^{i \theta}\right)}{\left(1-z r e^{-i \theta}\right)^{2}} d \theta d r \\
& =: F_{1, n}(z)+F_{2, n}(z)+F_{3, n}(z)+F_{4, n}(z)=F_{n}(z) .
\end{aligned}
$$

Let us for example consider $F_{2, n}(z)$. Performing the differentiation, using (2.4) for $f$ and its derivative, and using $\left|1-z r e^{-i \theta_{n}^{\prime}}\right| \geq C|1-z \bar{\zeta}|$ for all $\zeta \in D$, all $D \in \mathcal{D}_{n}$, in the denominator, we get

$$
\left|\partial_{r} \frac{f\left(r e^{i \theta_{n}^{\prime}}\right)}{\left(1-z r e^{\left.-i \theta_{n}^{\prime}\right)^{2}}\right.}\right| \leq \frac{C}{\left|D_{n}\right|} \sum_{D \in \mathcal{D}_{n}} \int_{D}\left(\frac{|f(\zeta)|}{|1-z \bar{\zeta}|^{3}}+\frac{\left|f^{\prime}(\zeta)\right|}{|1-z \bar{\zeta}|^{2}}\right) d A(\zeta)
$$

Using $\left|r_{n}^{\prime}-r_{n}\right| \leq C|1-z \bar{\zeta}|$, and $\left|r_{n}^{\prime}-r_{n}\right| \leq C\left(1-|\zeta|^{2}\right)$ we can evaluate $F_{2, n}$ :

$$
\left|F_{2, n}(z)\right| \leq \int_{r_{n}}^{r_{n}^{\prime}}\left|\int_{r_{n}}^{r} \int_{\theta_{n}}^{\theta_{n}^{\prime}} a\left(\varrho e^{i \varphi}\right) \varrho d \varphi d \varrho\right|\left|\partial_{r} \frac{f\left(r e^{i \theta_{n}^{\prime}}\right)}{\left(1-z r e^{-i \theta_{n}^{\prime}}\right)^{2}}\right| d r
$$




$$
\begin{aligned}
& \leq C \int_{r_{n}}^{r_{n}^{\prime}}\left|\int_{r_{n}}^{r} \int_{\theta_{n}}^{\theta_{n}^{\prime}} a\left(\varrho e^{i \varphi}\right) \varrho d \varphi d \varrho\right| \frac{1}{\left|D_{n}\right|} \sum_{D \in \mathcal{D}_{n}} \int_{D}\left(\frac{|f(\zeta)|}{|1-z \bar{\zeta}|^{3}}+\frac{\left|f^{\prime}(\zeta)\right|}{|1-z \bar{\zeta}|^{2}}\right) d A(\zeta) d r \\
& \leq C^{\prime} \sum_{D \in \mathcal{D}_{n}} \int_{r_{n}}^{r_{n}^{\prime}} \int_{D}\left(\frac{|f(\zeta)|}{|1-z \bar{\zeta}|^{3}}+\frac{\left|f^{\prime}(\zeta)\right|}{|1-z \bar{\zeta}|^{2}}\right) d A(\zeta) d r \\
& \leq C^{\prime \prime} \sum_{D \in \mathcal{D}_{n}} \int_{D}\left(\frac{|f(\zeta)|}{|1-z \bar{\zeta}|^{2}}+\frac{\left|f^{\prime}(\zeta)\right|\left(1-|\zeta|^{2}\right)}{|1-z \bar{\zeta}|^{2}}\right) d A(\zeta) .
\end{aligned}
$$

where the bound for the integral of $a$ follows from (1.4).

The other terms with $j=1,3,4$ can be estimated analogously, with a second derivative of $f$ in the case of $j=4$. Thus,

$$
\begin{aligned}
\left|F_{n}(z)\right| & \leq C \sum_{D \in \mathcal{D}_{n}} G_{D}(z), \text { where } \\
G_{D}(z) & =\int_{D}\left(\frac{|f(\zeta)|}{|1-z \bar{\zeta}|^{2}}+\frac{\left|f^{\prime}(\zeta)\right| W(\zeta)}{|1-z \bar{\zeta}|^{2}}+\frac{\left|f^{\prime \prime}(\zeta)\right| W(\zeta)^{2}}{|1-z \bar{\zeta}|^{2}}\right) d A(\zeta) .
\end{aligned}
$$

By Theorem 4.28 of [17], the function $g:=|f|+\left|f^{\prime}\right| W+\left|f^{\prime \prime}\right| W^{2}$ in the integrand belongs to $L^{p}$, and $\|g\|_{p} \leq C\|f\|_{p}$. Since $\cup_{n \in \mathbb{N}} D_{n}=\mathbb{D}$, we have

$$
\sum_{n=1}^{\infty} G_{D_{n}}(z)=\int_{\mathbb{D}}\left(\frac{|f(\zeta)|}{|1-z \bar{\zeta}|^{2}}+\frac{\left|f^{\prime}(\zeta)\right| W(\zeta)}{|1-z \bar{\zeta}|^{2}}+\frac{\left|f^{\prime \prime}(\zeta)\right| W(\zeta)^{2}}{|1-z \bar{\zeta}|^{2}}\right) d A(\zeta)
$$

notice that the series on the left has positive terms and the right hand side is the maximal Bergman projection $|P| g \in L^{p}$ of $g \in L^{p}$. In particular, the series on the left of (2.8) converges for almost all $z$, and the same is thus true for

$$
\sum_{n=1}^{\infty} \sum_{D \in \mathcal{D}_{n}} G_{D}(z)
$$

since the terms of (2.9) consist of the positive expressions $G_{D_{n}}$, and any single $G_{D_{n}}$ can occur at most $M N$ times in (2.9), by the definition of the numbers $N$ and $M$.

By (2.7), the convergence of (2.9) implies the absolute convergence of the series $\sum_{n} F_{n} f(z)$ a.e.. We claim that the operator sequence $\left(T^{(m)}\right)_{m=1}^{\infty}$ defined by

$$
T^{(m)} f=\sum_{n=1}^{m} F_{n} f
$$

converges to $\mathbf{T}_{a}$ in the SOT, as $m \rightarrow \infty$. Indeed, given $f \in A^{p}$ and any $z \in \mathbb{D}$, the difference

$$
\left|\mathbf{T}_{a} f(z)-T^{(m)} f(z)\right|=\left|\sum_{n>m} F_{n} f(z)\right|=\left|\int_{V_{m}} \frac{a(\zeta) f(\zeta)}{(1-z \bar{\zeta})^{2}} d A(\zeta)\right|,
$$

where $V_{m}=\cup_{n>m} D_{n}$, has by (2.7) the upper bound

$$
C \int_{V_{\mu}} \frac{g(\zeta)}{\mid 1-z \bar{\zeta})^{2} \mid} d A(\zeta)=C \int_{\mathbb{D}} \frac{\chi_{V_{\mu}}(\zeta) g(\zeta)}{|1-z \bar{\zeta}|^{2}} d A(\zeta)=C|P|\left(\chi_{V_{\mu}} g\right)(z)
$$


here, $g$ is as below $(2.7), \mu$ is some positive integer with $\mu \rightarrow \infty$ as $m \rightarrow \infty$, and $\chi_{V_{\mu}}$ is the characteristic function of the set $V_{\mu}$. But we have $\left\|\chi_{V_{\mu}} g\right\|_{p} \rightarrow 0$ as $\mu \rightarrow \infty$, by Lebesgue's dominated convergence theorem. Since $|P|$ is a bounded operator, there also holds $\left\||P|\left(\chi_{V_{\mu}} g\right)\right\|_{p} \rightarrow 0$ as $\mu \rightarrow \infty$. Combining this with the estimates (2.11)-(2.12) we get that $\left\|\mathbf{T}_{a} f-T^{(m)} f\right\|_{p} \rightarrow 0$ as $m \rightarrow \infty$, which proves the claim.

(ii) We next consider the relation of the limit in (2.2) with the sum (1.8).

Let us fix $n$ for a moment. Arguing as in (2.5)-(2.7), with straightforward changes of the upper ends of the integration intervals, we see that given any $(\tilde{r}, \tilde{\theta})$ such that $r_{n}<\tilde{r}<r_{n}^{\prime}$ and $\theta_{n}<\tilde{\theta}<\theta_{n}^{\prime}$, the expression

$$
G_{n}(z, \tilde{r}, \tilde{\theta}):=\int_{r_{n}}^{\tilde{r}} \int_{\theta_{n}}^{\tilde{\theta}} \frac{a\left(\varrho e^{i \varphi}\right) f\left(\varrho e^{i \varphi}\right)}{\left(1-z \varrho e^{-i \varphi}\right)^{2}} \varrho d \varrho d \varphi
$$

has the same upper bound as $F_{n} f(z)$ in (2.7) (cf. (1.7)), namely

$$
\left|G_{n}(z, \tilde{r}, \tilde{\theta})\right| \leq C \sum_{D \in \mathcal{D}_{n}} G_{D}(z) .
$$

Given $\rho, 0<\rho<1$, the integral in (2.2) can be written as

$$
\int_{\mathbb{D}_{\rho}} \frac{a(\zeta) f(\zeta)}{(1-z \bar{\zeta})^{2}} d A(\zeta)=\sum_{n=1}^{m} F_{n} f(z)+\sum_{n=m+1}^{K} G_{n}\left(z, \rho, \theta_{n}^{\prime}\right)
$$

for some integers $m$ and $K>m$, and moreover, $m \rightarrow \infty$ as $\rho \rightarrow 1$. It is then obvious from the estimate (2.13) and the convergence of (2.9) that for almost all $z$, the limit in (2.2) must exist and, by (2.14), it has to coincide with $\sum_{n} F_{n} f(z)=\mathbf{T}_{a} f(z)$, (1.8).

Concerning the convergence in the SOT, we use (2.13) and (2.14) and the argument around (2.11)-(2.12) to estimate the difference

$$
\left|\mathbf{T}_{a} f(z)-T_{a_{\rho}} f(z)\right| \leq C|P|\left(\chi_{V_{\mu}} g\right)(z)
$$

where $\mu \rightarrow \infty$ as $\rho \rightarrow 1$. Convergence in the SOT follows in the same way as at the end of part $(i)$.

(iii) Let us consider (2.3); let $f \in A^{p}$ and $g \in A^{q}$ be given. Denoting by $\langle\cdot, \cdot\rangle$ the standard complex dual paring of $A^{p}$ and $A^{q}$, we have

$$
\left\langle f, \mathbf{T}_{a}^{*} g\right\rangle=\left\langle\mathbf{T}_{a} f, g\right\rangle=\int_{\mathbb{D}} \bar{g} \lim _{\rho \rightarrow 1} T_{a_{\rho}} f d A=\lim _{\rho \rightarrow 1} \int_{\mathbb{D}} \bar{g} T_{a_{\rho}} f d A,
$$

where the limit and the integral could be commuted because of the convergence of (2.2) in the SOT. Then, (2.16) equals

$$
\begin{aligned}
& \lim _{\rho \rightarrow 1} \int_{\mathbb{D}} \int_{\mathbb{D}_{\rho}} \frac{a(\zeta) f(\zeta)}{(1-z \bar{\zeta})^{2}} d A(\zeta) \overline{g(z)} d A(z) \\
= & \lim _{\rho \rightarrow 1} \int_{\mathbb{D}} f(\zeta) a_{\rho}(\zeta) \overline{P g(\zeta)} d A(\zeta)=\lim _{\rho \rightarrow 1} \int_{\mathbb{D}} f(\zeta) a_{\rho}(\zeta) \overline{g(\zeta)} d A(\zeta) \\
= & \lim _{\rho \rightarrow 1} \int_{\mathbb{D}} f(\zeta) \overline{P\left(\overline{a_{\rho}} g\right)(\zeta)} d A(\zeta)=\lim _{\rho \rightarrow 1} \int_{\mathbb{D}} f \overline{T_{\bar{a}_{\rho}} g} d A=\int_{\mathbb{D}} f \lim _{\rho \rightarrow 1} \overline{T_{\bar{a}_{\rho}} g} d A,
\end{aligned}
$$


where at the end we used the fact that $\bar{a}$ obviously also satisfies condition (1.4) and the convergence of (2.2) in the SOT.

That the limit exist in the SOT follows from the treatment of the limit (2.2), since $\bar{a}$ satisfies (1.4). The proof of the little Hankel case is similar, with obvious changes.

\section{Concluding Remarks.}

We have the following consequence concerning generalized Toeplitz and little Hankel operators.

Corollary 3.1. Let $1<p<\infty$ and assume that (1.4) holds for the symbol $a \in L_{\text {loc }}^{1}$. Let $f \in A^{p}$ be such that af $\in L^{1}$. Then,

$$
\mathbf{T}_{a} f(z)=\int_{\mathbb{D}} \frac{a(\zeta) f(\zeta)}{(1-z \bar{\zeta})^{2}} d A(\zeta) \text { and } \mathbf{h}_{a} f(z)=\int_{\mathbb{D}} \frac{a(\zeta) f(\zeta)}{(1-\bar{z} \zeta)^{2}} d A(\zeta)
$$

for all $z \in \mathbb{D}$.

Proof. In the case of the Toeplitz operator we have the integral representation (2.1) for $T_{a_{\rho}}$ with any $0<\rho<1$. The integral formula (3.1) follows by applying Lebesgue's dominated convergence theorem in the limit (2.2), since we have af $\in L^{1}$ by assumption. The proof in the case of little Hankel operators is the same.

The sufficient condition (1.4) and the definitions (1.8), (2.2) of Toeplitz operators are formulated for quite general locally integrable symbols, but the following example shows that the condition and the boundedness result are useful already in very simple, concrete cases. A well known sufficient condition for the boundedness of $T_{a}$ is that

$$
\sup _{D \in \mathcal{D}} M_{a}(D)<\infty \text { where } M_{a}(D):=\frac{1}{|D|} \int_{D}|a| d A,
$$

and this condition is also necessary, if $\mathbb{R} \ni a(z) \geq 0$ for all $z \in \mathbb{D}$. See [17].

For every $0<b \leq 1 / 2$ we define the symbol

$$
a_{b}\left(r e^{i \theta}\right):= \begin{cases}\frac{1}{r(1-r)^{b}} \sin \frac{1}{1-r}, & r \geq \frac{1}{2} \\ 1, & r<\frac{1}{2}\end{cases}
$$

which obviously belongs to $L^{q}$, if $b q<1$. Then, in particular, $a_{1 / 4} \in L^{2}$ and the defining integral formula of $T_{a_{1 / 4}}$ converges for every $f \in A^{2}$. Obviously, the defining formula of $T_{\left|a_{1 / 4}\right|}$ also converges for every $f \in A^{2}$. However, we have the following result.

Proposition 3.2. ( $i)$ The Toeplitz operator $T_{\left|a_{b}\right|}$ is not bounded in $A^{p}$ for any $1<$ $p<\infty$ and $0<b \leq 1 / 2$.

(ii) The Toeplitz operator $T_{a_{b}}$ is bounded in $A^{p}$ for all $1<p<\infty$ and $0<b \leq 1 / 2$.

Proof. Let us first deal with $T_{\left|a_{b}\right|}$. Given $0<r<1$ and any $\theta \in[0,2 \pi]$, we consider the behaviour of $a_{b}$ in the set $D=D(r, \theta)$, see Definition 1.1. It is plain 
from the definition of $a_{b}$ and the elementary properties of the sinus that for some universal constant $C>0$ we have

$$
\left|a_{b}(z)\right| \geq \frac{1}{4}(1-|z|)^{-b}
$$

in a subset of $D$ with area measure at least $C(1-r)^{2}$ (recall that $|D|$ is proportional to $\left.(1-r)^{2}\right)$. Then, of course $M_{a}(D) \geq C^{\prime}(1-r)^{-b}$ for another constant $C^{\prime}>0$, and thus condition (3.2) cannot hold, and the operator $T_{\left|a_{b}\right|}$ is unbounded.

The symbol satisfies (1.4), since given $D=D(1-2 \delta, \theta)$ with a small enough $\delta$ and $\zeta=\rho e^{i \phi} \in D$, we have, using the change of variable $y=1 /(1-\varrho)$ (so that $\left.d \varrho=y^{-2} d y\right)$

$$
|D|\left|\hat{a}_{D}(\zeta)\right|=\int_{\theta}^{\phi} d \varphi\left|\int_{1-2 \delta}^{\rho} \frac{1}{(1-\varrho)^{b}} \sin \frac{1}{1-\varrho} d \varrho\right|=\pi \delta\left|\int_{1 /(2 \delta)}^{1 /(1-\rho)} y^{b-2} \sin y d y\right|
$$

Let us divide the integration interval to subintervals $J_{n}:=[2 \pi n, 2 \pi(n+1)], n \in \mathbb{N}$. On $J_{n}$ we integrate as follows:

$$
\begin{aligned}
& \left|\int_{J_{n}} y^{b-2} \sin y d y\right|=\left|\int_{J_{n}} y^{b-2} \sin y d y-(2 \pi(n+1))^{b-2} \int_{J_{n}} \sin y d y\right| \\
\leq & \int_{J_{n}}\left|y^{b-2}-(2 \pi(n+1))^{b-2}\right| d y \leq C n^{b-3} .
\end{aligned}
$$

Hence,

$$
|D|\left|\hat{a}_{D}(\zeta)\right| \leq \pi \delta\left|\int_{1 /(2 \delta)}^{1 /(1-\rho)} y^{b-2} \sin y d y\right| \leq C \delta \sum_{n=[1 /(4 \pi \delta)]}^{\infty} n^{b-3} \leq C^{\prime} \delta^{3-b},
$$

where $[x]$ denotes the integer part of a number $x \in \mathbb{R}$. Since $|D|$ is proportional to $\delta^{2}$, the condition (1.4) holds true, and $T_{a_{b}}$ is bounded, by Theorem 1.2 and Corollary 3.1 .

Acknowledgements. The authors wish to thank Grigori Rozenblum (Göteborg) for some personal communication which initiated the investigation leading to this work. The authors are also grateful for the anonymous referees for remarks that helped to improve the results of this paper.

The research of JT was partially supported by the Väisälä Foundation of the Finnish Academy of Science and Letters. The research of JV and the visit of JT to the University of Reading were supported by EPSRC grant EP/M024784/1.

\section{REFERENCES}

[1] M. Engliš, Toeplitz operators and weighted Bergman kernels. J. Funct. Anal. 255, 6 (2008), 1419-1457.

[2] S. Grudsky, A. Karapetyants, N. Vasilevski, Toeplitz operators on the unit ball in $\mathbb{C}^{N}$ with radial symbols, J. Oper. Theory 49 (2003), 325-346.

[3] S. Grudsky, N. Vasilevski, Bergman-Toeplitz operators: radial component influence, Integr. Eq. Oper. Theory 40 (2001), 16-33.

[4] D. H. Luecking, Trace ideal criteria for Toeplitz operators. J. Funct. Anal. 73 (1987), no. 2, 345-368. 
[5] D. H. Luecking, Finite rank Toeplitz operators on the Bergman space. Proc. Amer. Math. Soc. 136 (2008), no. 5, 1717-1723.

[6] W. Lusky, J. Taskinen, Toeplitz operators on Bergman spaces and Hardy multipliers. Studia Math. 204 (2011), 137-154.

[7] P. Mannersalo, Toeplitz operators with locally integrable symbols on Bergman spaces of bounded simply connected domains, Compl.Variables Elliptic Eq. 61,6 (2016), 854-874.

[8] A. Perälä, J. Taskinen, and J. A. Virtanen, Toeplitz operators with distributional symbols on Bergman spaces, Proc. Edinb. Math. Soc. 54, 2 (2011), 505-514.

[9] W. Rudin, Real and Complex analysis, 3rd ed., McGraw-Hill, New York, 1987.

[10] K. Stroethoff, Compact Toeplitz operators on Bergman spaces, Math. Proc. Cambridge Philos. Soc. 124(1998), 151-160.

[11] K. Stroethoff, D. Zheng, Toeplitz and Hankel operators on Bergman spaces, Trans. AMS 329, 2 (1992), 773-794.

[12] D. Suárez, The essential norm of operators in the Toeplitz algebra on $A^{p}\left(B_{n}\right)$, Indiana Univ. Math. J. 56, no. 5, (2007) 2185-2232.

[13] J. Taskinen, J. A. Virtanen, Toeplitz operators on Bergman spaces with locally integrable symbols. Rev.Math.Iberoamericana 26,2 (2010), 693-706.

[14] N.L. Vasilevski, Bergman type spaces on the unit disk and Toeplitz operators with radial symbols, Reporte Interno 245, Departamento de Matemáticas, CINVESTAV del I.P.N., Mexico City, 1999.

[15] N.L. Vasilevski, Commutative algebras of Toeplitz operators on the Bergman space, Operator Theory: Advances and Applications, Vol. 185, Birkhäuser Verlag, 2008.

[16] K. Zhu, Positive Toeplitz operators on weighted Bergman space, J. Operator Theory 20 (1988), 329-357.

[17] K. Zhu, Operator Theory in Function Spaces, 2nd edition, Mathematical Surveys and Monographs, 138, American Mathematical Society, Providence, RI, 2007.

[18] N. Zorboska, Toeplitz operators with BMO symbols and the Berezin transform. Int. J. Math. Math. Sci. 46 (2003), 2929-2945.

Department of Mathematics, University of Helsinki, 00014 Helsinki, Finland

E-mail address: jari.taskinen@helsinki.fi

Department of Mathematics, University of Reading, Whiteknights, P.O. Box 220, READING RG6 6AX, UK

E-mail address: j.a.virtanen@reading.ac.uk 